\title{
Comparison of analgesic efficacy between rectus sheath blockade, intrathecal morphine with bupivacaine, and intravenous patient-controlled analgesia in patients undergoing robot-assisted laparoscopic prostatectomy: a prospective, observational clinical study
}

Jung-Woo Shim', Yun Jeong Cho' ${ }^{1}$, Minhee Kim², Sang Hyun Hong ${ }^{1}$, Hyong Woo Moon ${ }^{3}$, Sung Hoo Hong ${ }^{3}$ and Min Suk Chae ${ }^{1 *}$ (D)

\begin{abstract}
Background: We explored the analgesic outcomes on postoperative day (POD) 1 in patients undergoing robotassisted laparoscopic prostatectomy (RALP) who received intravenous patient-controlled analgesia (IV-PCA), rectus sheath bupivacaine block (RSB), or intrathecal morphine with bupivacaine block (ITMB).

Methods: This was a prospective, observational clinical trial. Patients were divided into three groups: IV-PCA $(n=30)$, RSB $(n=30)$, and ITMB $(n=30)$. Peak pain scores at rest and with coughing, cumulative IV-PCA drug consumption, the need for IV rescue opioids, and Quality of Recovery-15 (QoR-15) questionnaire scores collected on POD 1 were compared among the groups.
\end{abstract}

\footnotetext{
* Correspondence: shscms@gmail.com
}

'Department of Anesthesiology and Pain medicine, Seoul St. Mary's Hospital, College of Medicine, The Catholic University of Korea, 222, Banpo-daero, Seocho-gu, Seoul 06591, Republic of Korea

Full list of author information is available at the end of the article

C C The Author(s). 2020 Open Access This article is licensed under a Creative Commons Attribution 4.0 International License, which permits use, sharing, adaptation, distribution and reproduction in any medium or format, as long as you give appropriate credit to the original author(s) and the source, provide a link to the Creative Commons licence, and indicate if changes were made. The images or other third party material in this article are included in the article's Creative Commons licence, unless indicated otherwise in a credit line to the material. If material is not included in the article's Creative Commons licence and your intended use is not permitted by statutory regulation or exceeds the permitted use, you will need to obtain permission directly from the copyright holder. To view a copy of this licence, visit http://creativecommons.org/licenses/by/4.0/. The Creative Commons Public Domain Dedication waiver (http://creativecommons.org/publicdomain/zero/1.0/) applies to the data made available in this article, unless otherwise stated in a credit line to the data. 
(Continued from previous page)

Results: The preoperative and intraoperative findings were comparable among the groups; the ITMB group required the least remifentanil of all groups. During POD 1, the ITMB group reported lower levels of pain at rest and with coughing, compared with the other two groups. During POD 1, incidences of severe pain at rest (10.0\% vs. 23.3\% vs. 40.0\%) and with coughing (16.7\% vs. $36.7 \%$ vs. 66.7\%) were the lowest in the ITMB group compared with the RSB and IV-PCA groups, respectively. After adjustment for age, body mass index, diabetes mellitus, hypertension, and intraoperative remifentanil infusion, severe pain at rest was 0.167-fold less common in the ITMB group than in the IV-PCA group, while pain with coughing was 0.1-fold lower in the ITMB group and 0.306-fold lower in the RSB group, compared with the IV-PCA group. The ITMB group required lower cumulative IV-PCA drug infusions and less IV rescue opioids, while exhibiting a better QoR-15 global score, compared with the other two groups. Complications (nausea and pruritus) were significantly more common in the ITMB group than in the other two groups; however, we noted no ITMB- or RSB-related anesthetic complications (respiratory depression, post-dural headache, nerve injury, or puncture site hematoma or infection), and all patients were assessed as Clavien-Dindo grade I or II during the hospital stay.

Conclusion: Although ITMB induced complications of nausea and pruritus, this analgesic technique provided appropriate pain relief that enhanced patient perception related to early postoperative recovery.

Trial registration: Clinical Research Information Service, Republic of Korea, (approval number: KCT0005040) on May 20, 2020

Keywords: Rectus sheath bupivacaine, Intrathecal morphine with bupivacaine, Robot-assisted laparoscopic prostatectomy

\section{Background}

Robot-assisted laparoscopic prostatectomy (RALP) is a technically advanced, minimally invasive surgical method that affords much better surgical view and greater maneuverability than open or laparoscopic prostatectomy [1]. Previous studies have found that RALP is associated with better oncological and functional outcomes, compared with open or laparoscopic radical prostatectomy $[2,3]$. However, RALP patients frequently experience unbearable pain that persists over several days after surgery and requires pain-relief medications, such as opioids. This pain arises from skin-port incisions, multiple dissections of prostateinvolved and surrounding tissues, bladder spasm, and transurethral catheter irritation [4]. Various central and/or peripheral pain-relief methods have been used to attenuate the severe pain that develops immediately after RALP $[5,6]$. A rectus sheath block (RSB) regimen affords peri-umbilical incision site analgesia superior to that achieved via local anesthetic infiltration; this site is the principal source of pain immediately after laparoscopy-based surgery [7]. Compared with transversus abdominis plane (TAP) block, RSB may afford better analgesia when a midline incision is created and more prolonged blockade of noxious input from that site [8]. An intrathecal morphine and bupivacaine block (ITMB) regimen affords pain relief for $20-48 \mathrm{~h}$ postoperatively and reduces bladder spasm-related discomfort (a common complication associated with urinary catheter insertion after prostate surgery) [9]. However, no ideal analgesic regimen has been described that affords maximal benefits with minimal side effects; this would improve the quality of early postoperative recovery after RALP.
The aim of this study was to evaluate the analgesic outcomes on postoperative day (POD) 1 in patients undergoing RALP who received RSB or ITMB, compared with patients who received intravenous patientcontrolled analgesia (IV-PCA) alone. We also compared postoperative complications and patient satisfaction. We hypothesized that, due to a reduction in surgical pain and bladder spasm-related discomfort, the ITMB regimen would lead to improved pain relief and satisfaction with recovery on the first day after surgery, compared with the other analgesic regimens.

\section{Methods}

\section{Ethical considerations}

This was a prospective, observational parallel-cohort trial. The protocol was approved by the Institutional Review Board of Seoul St. Mary's Hospital Ethics Committee (approval no. KC20OISI0124) on April 29, 2020. The study was performed in accordance with all relevant principles of the Declaration of Helsinki. The study protocol was prospectively registered on a publicly accessible clinical registration site recognized by the International Committee of Medical Journal Editors (Clinical Research Information Service, Republic of Korea; approval no. KCT0005040) on May 20, 2020. Written informed consent was obtained from all patients enrolled between May 2020 and July 2020. The study adhered to Strengthening the Reporting of Observational Studies in Epidemiology guidelines (Additional file 1); a study flow chart is shown in Fig. 1.

\section{Study population}

The inclusion criteria for our study were: age 19-74 years, prostate cancer stage I or II [10], patients 


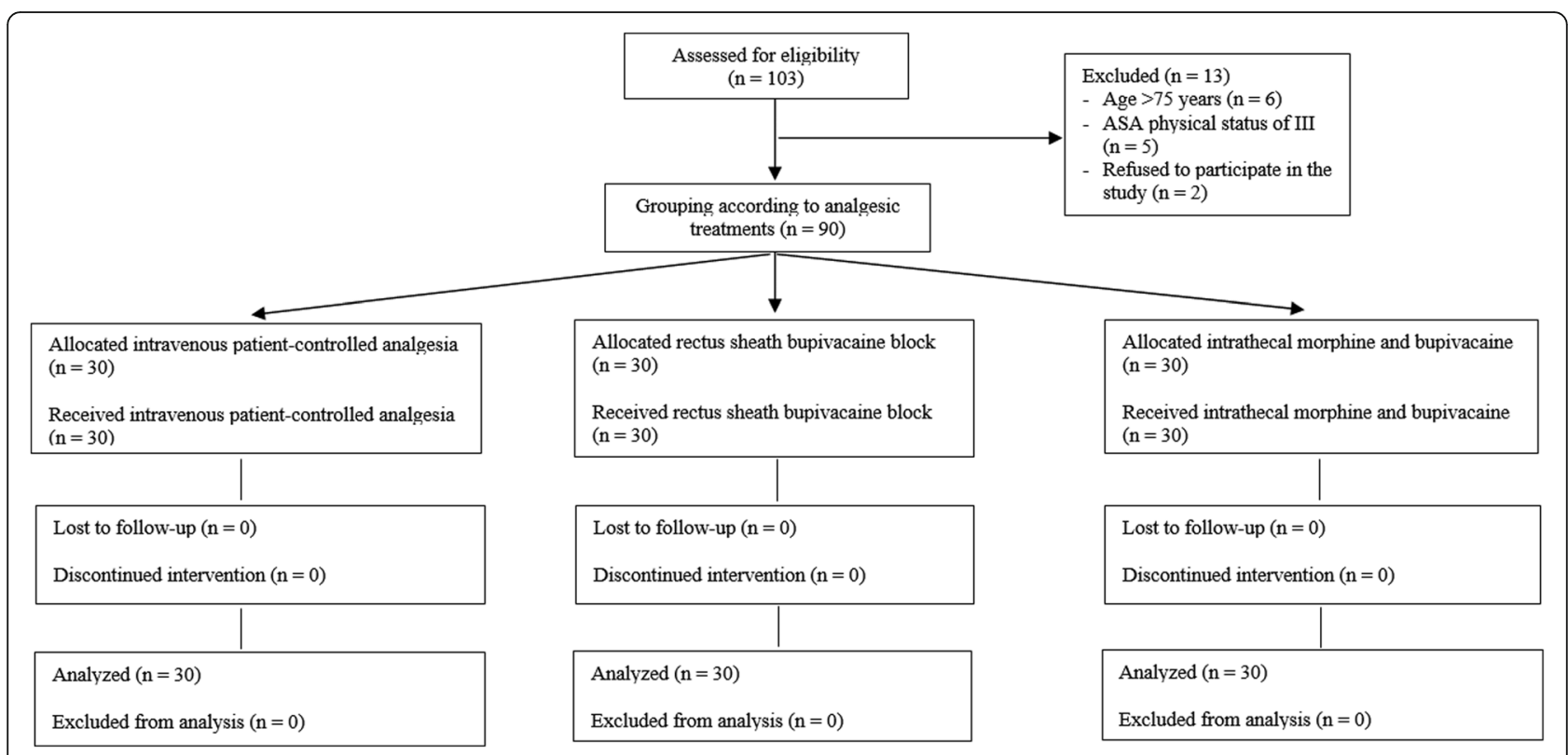

Fig. 1 Flow chart of the study

scheduled for elective RALP, and American Society of Anesthesiologists (ASA) physical status I or II. The exclusion criteria were: a history of allergy to a local anesthetic or opioid drug, coagulopathy (international normalized ratio [INR] $>1.5$ for ITMB or INR $>2.0$ for a single injection of ultrasound-guided RSB; and platelet count $\left.<100.0 \times 10^{9} / \mathrm{L}\right)[11,12]$, hemodynamic instability that required strong vasopressors (i.e., epinephrine or norepinephrine), hetastarch colloid infusion, or blood product transfusion (i.e., packed red blood cells $\geq 1$ unit due to hemoglobin $<7.0 \mathrm{~g} / \mathrm{dL}$ ) [13], and refusal to participate.

The patients were divided into three groups based on their analgesia preference: IV-PCA alone (reference group), RSB and IV-PCA (RSB group), and ITMB and IV-PCA (ITMB group).

\section{Patient management in the operating room}

The RALP surgical technique and balanced anesthetic management were as described previously [14]; patient care was standardized apart from the analgesic treatments. Briefly, balanced anesthesia was performed by attending expert anesthesiologists. Induction of anesthesia was achieved using $1-2 \mathrm{mg} / \mathrm{kg}$ of propofol and $0.6 \mathrm{mg} /$ $\mathrm{kg}$ of rocuronium; anesthesia was then maintained using $2.0-6.0 \%$ desflurane under medical air in oxygen. Remifentanil was continuously infused at a rate of $0.1-0.5 \mu \mathrm{g} /$ $\mathrm{kg} / \mathrm{min}$, as appropriate. The Bispectral Index ${ }^{\mathrm{Tm}}$ instrument was set between 40 and 50 to ensure appropriate hypnotic depth. Rocuronium was repeatedly infused under train-of-four monitoring ( $>1$ twitch). End-tidal $\mathrm{CO}_{2}$ was set between 30 and $40 \mathrm{mmHg}$ with adjustment of the ventilator mode. For fluid therapy, a baseline isotonic crystalloid was prepared based on the estimated fluid maintenance requirements, which were established in accordance with the patient's weight and anticipated tissue trauma. Additional fluid boluses were infused according to blood loss; however, the total amount of fluid was restricted to a maximum of $1 \mathrm{~L}$ before vesicourethral anastomosis.

The attending anesthesiologists (whose subspecialty involved regional blocks) and nurses were aware of the group allocations, but were not involved in later patient care or data collection (other than the completion of medical records). RSB was established immediately after the induction of general anesthesia. An ultrasound probe was positioned transversely on the rectus abdominis muscle, above the umbilicus (Fig. 2). Guided by realtime ultrasound, a sterile 22-G Tuohy-type epidural needle was cautiously advanced in-plane (to prevent injury to nearby vessels) from medially to laterally until the tip attained the plane between the lateral side of the rectus abdominis muscle and the posterior rectus sheath. After negative pressure aspiration, $20 \mathrm{~mL}$ of $0.25 \%(\mathrm{w} / \mathrm{v})$ bupivacaine was administered and the block was repeated on the opposite side. ITMB was placed before the induction of general anesthesia. Each patient received $0.2 \mathrm{mg}$ of intrathecal morphine sulfate and $7.5 \mathrm{mg}$ of bupivacaine by means of a sterile 25-G Quincke-type spinal needle inserted between lumbar vertebrae 3 and 4 . The drugs were administered through a single injection after collection of cerebrospinal fluid. All patients were allowed access to IV-PCA (1000 $\mu \mathrm{g}$ of fentanyl, $90 \mathrm{mg}$ of ketorolac, and $0.3 \mathrm{mg}$ of ramosetron). The IV-PCA regimen featured 


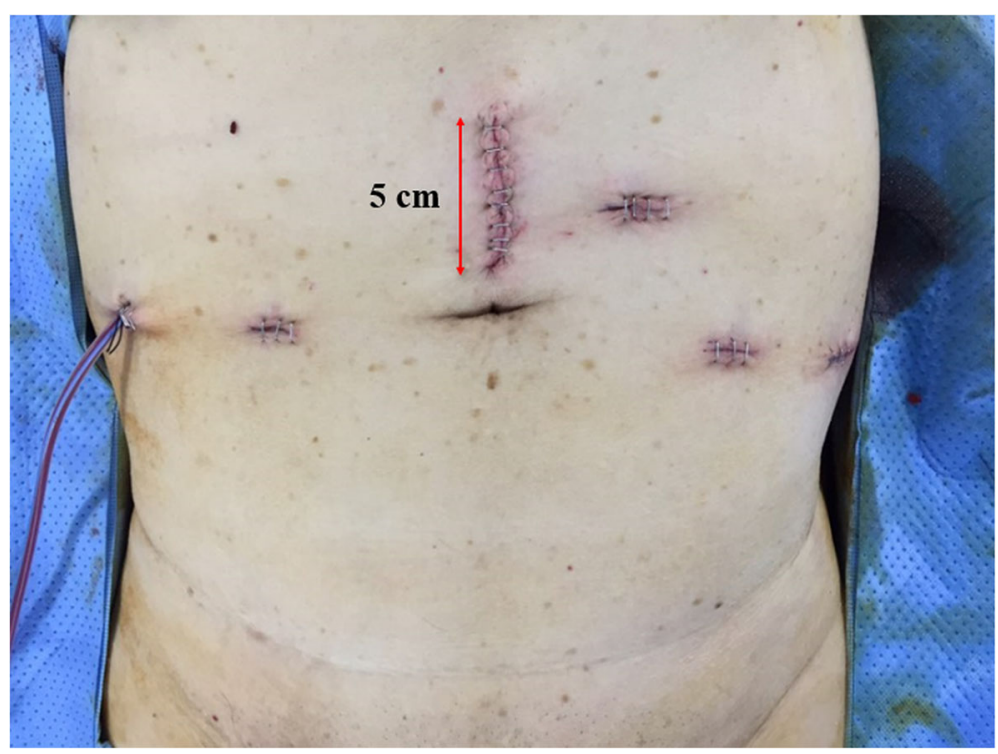

Fig. 2 Peri-umbilical wound site (arrow) that is the principal analgesic target of RSB

a 2-mL bolus injection and $0.5 \mathrm{~mL} / \mathrm{h}$ basal infusion with a lockout time of $10 \mathrm{~min}$. If a patient experienced acute postoperative breakthrough pain (visual analog scale [VAS] score $\geq 7$ ), $25 \mathrm{mg}$ of pethidine (an IV rescue opioid) was administered based on the discretion of the attending physicians (in the postoperative acute care unit or ward), who were blinded to group assignment.

\section{Pain outcomes}

Cumulative IV-PCA drug consumption and the need for IV rescue opioids were primarily assessed during the first $24 \mathrm{~h}$ postoperatively. Peak pain scores at rest and with coughing were assessed using a VAS that ranged from 0 to 10 , where " 0 " represented no pain and " 10 " represented the worst possible pain. Pain severity was classified as mild (VAS scores 0-3), moderate (4-6), or severe (7-10) [15]. Pain was assessed using the VAS three times (i.e., at $1 \mathrm{~h}$ postoperatively in the postanesthesia care unit [PACU], as well as at 6 and $24 \mathrm{~h}$ postoperatively in the ward). If a patient experienced acute postoperative breakthrough pain (VAS score $\geq 7$ ), $25 \mathrm{mg}$ of pethidine (an IV rescue opioid) was administered by attending physicians (anesthesiologists in the PACU and urologists in the ward) or nurses, none of whom were aware of the group allocations.

\section{Clinical variables}

Preoperative demographic and laboratory parameters were recorded on the day before surgery by attending urologic physicians or nurses in the ward, who were not aware of the group allocations and were not involved in further data collection other than filling in medical record forms. Intraoperative findings, such as surgical duration, hypotension status (systolic blood pressure $<90 \mathrm{mmHg}$ for more than $10 \mathrm{~min}$ ), total rescue ephedrine infusion, total remifentanil infusion, crystalloid fluid infusion, urine output, and hemorrhage status, were recorded by the attending anesthesiologists or nurses in the operating room, who were not involved in further patient care or data collection (other than filling in anesthetic record forms) and were not aware of the group allocations. Postoperative findings, such as the global quality-ofrecovery score on a 15-item questionnaire (the QoR15) [16]; the incidences of nausea, vomiting, and pruritus; the Clavien-Dindo grade [17]; and laboratory variables, were measured in the ward on the first day after surgery (between 6 and $8 \mathrm{pm}$ ). These findings were measured by anesthesiology residents (Y.J.C. and M.K.) who were not aware of the group allocations and were not involved in further patient care or data collection. Additionally, nausea and vomiting were assessed on a binary scale (yes/no). Patients were considered to have nausea, if they responded positively to the question, "are you or have you felt nauseated after surgery?". Using similar questions, vomiting episodes were assessed [18]. Pruritus was assessed using the following scale: $0=$ no itch; $1=i$ itch with no need to scratch, just rubbing (mild); $2=$ itch with need to scratch (moderate); $3=$ itch with need to scratch and requiring treatment (severe) [19]. We defined overt pruritus as a score $\geq 2$. Therefore, nausea, vomiting, and pruritus were considered binary variables in our analysis. 


\section{Statistical analyses}

The minimum sample size was based on the difference in cumulative IV-PCA drug consumption on POD 1 between patients who received RSB and those who received ITMB, calculated using electronic medical records. Mean cumulative IV-PCA drug consumption on POD 1 by patients who received RSB $(n=10)$ and those who received ITMB $(n=10)$ were 47.1 and $29.1 \mathrm{~mL}$, respectively. The standard deviation (SD) among the 20 patients was $23.6 \mathrm{~mL}$. Therefore, a minimum sample size of 27 patients/group was required to afford an $\alpha$ value of 0.05 and a power of 0.8 . We recruited 30 patients for each group; we assumed a dropout rate of $10 \%$.

Data are expressed as means \pm standard deviations (SDs), medians with interquartile ranges (IQRs), or numbers with proportions (\%), as appropriate. The normality of continuous data distributions was evaluated using the Shapiro-Wilk test. Continuous perioperative variables of the three groups were compared via one-way analysis of variance or the Kruskal-Wallis test; post hoc testing employed the unpaired $t$-test or the Mann-Whitney $U$ test. Perioperative categorical variables were compared among the groups using the Pearson $\chi^{2}$ test or Fisher's exact test, as appropriate. Trend testing employed a linear-by-linear association method. To determine the clinical analgesic efficacy of the treatments, logistic regression analysis was used to derive odds ratios with 95\% confidence intervals of the risks (postoperative peak VAS score $\geq 7$ at rest and with coughing) associated with IV-PCA alone (reference), and the RSB and ITMB, after adjusting for age, body mass index, and diabetes mellitus and hypertension statuses (these comorbidities may change in accordance with pain level) [20, 21], and intraoperative remifentanil consumption. Tests for linear trends among patients in terms of cumulative IV-PCA drug consumption on POD 1 were based on stepwise linear regression. All tests were two-sided and a $p$-value $<0.017$ was considered statistically significant (multiple comparisons were made). All statistical analyses were performed with the aid of SPSS for Windows (ver. 24.0; IBM Corp., Armonk, NY, USA) and MedCalc for Windows (ver. 11.0; MedCalc Software, Ostend, Belgium).

\section{Results}

\section{Study population}

In total, 103 patients were assessed for eligibility. Thirteen patients were excluded: six were aged $>74$ years, five had ASA physical status III, and two refused to participate. Thus, 90 patients were enrolled and divided into the IVPCA, RSB, and ITMB groups ( $n=30$ patients per group).

\section{Preoperative and intraoperative findings}

Of all patients $(n=90)$, the median age was $65(62-71)$ years and the median body mass index was 24.0 (22.5-
26.5) $\mathrm{kg} / \mathrm{m}^{2}$. In total, 15 patients (16.7\%) had diabetes mellitus and 35 (38.9\%) hypertension. None of the patients showed coagulopathic findings preoperatively; the minimum and maximum INRs were 0.8 and 1.0, while the minimum and maximum platelet counts were $120.0 \times 10^{9} / \mathrm{L}$ and $355.0 \times 10^{9} / \mathrm{L}$. Table 1 shows that the preoperative and intraoperative findings were comparable among the three groups. However, during surgery, the ITMB group exhibited the lowest remifentanil consumption, whereas the RSB group required less remifentanil than the IV-PCA group.

\section{Postoperative pain}

During POD 1, the ITMB group reported lower pain levels at rest and with coughing than did the RSB and IV-PCA groups (Fig. 3). After adjustment for age, body mass index, comorbidity status, and intraoperative remifentanil infusion, severe pain at rest was 0.167 -fold less common in the ITMB group than in the IV-PCA group, while pain with coughing was 0.1 -fold lower in the ITMB and 0.306-fold lower in the RSB group, compared with the IV-PCA group (Table 2). Table 3 shows that cumulative IV-PCA drug consumption decreased according to analgesic treatment in the following order: IV-PCA alone > RSB $>$ ITMB (linear regression, $p<$ 0.001). The ITMB group required less IV-PCA drug infusion and IV rescue opioids than did the RSB and IVPCA groups. The ITMB group had the lowest peak VAS scores at rest and with coughing, compared with the other two groups, while the RSB group had a lower peak VAS score with coughing, compared with the IV-PCA group (Table 4).

\section{Postoperative clinical findings}

The global QoR-15 questionnaire score was higher in the ITMB group than in the RSB and IV-PCA groups (Table 4). Complications (nausea and pruritus) were significantly more common in the ITMB group than in the other two groups; however, we noted no ITMB- or RSBrelated anesthetic complications (respiratory depression, post-dural headache, nerve injury, or puncture site hematoma or infection), and all patients were assessed as Clavien-Dindo grade I or II during the hospital stay.

\section{Discussion}

Our principal findings were that ITMB may afford superior analgesia and better patient perception of outcome in terms of early postoperative recovery, compared with RSB or IV-PCA alone, in patients undergoing RALP. The analgesic efficacy of ITMB was approximately three-fold and 10-fold better in terms of reducing severe pain during the early postoperative period, compared with RSB and IV-PCA alone, respectively. Although ITMB was associated with more nausea and 
Table 1 Comparisons of preoperative and intraoperative findings between the three groups

\begin{tabular}{|c|c|c|c|c|}
\hline Group & IV-PCA & RSB & ITMB & \\
\hline $\mathbf{n}$ & 30 & 30 & 30 & $p$ \\
\hline \multicolumn{5}{|l|}{ Preoperative findings } \\
\hline Age (years) & $65(61-69)$ & $67(64-72)$ & $65(62-71)$ & 0.286 \\
\hline Body mass index $\left(\mathrm{kg} / \mathrm{m}^{2}\right)$ & $24.4(22.7-27.7)$ & $23.6(22.2-25.2)$ & $24.2(22.3-26.4)$ & 0.276 \\
\hline \multicolumn{5}{|l|}{ Comorbidity } \\
\hline Hypertension & $9(30.0 \%)$ & $10(33.3 \%)$ & $16(53.3 \%)$ & 0.134 \\
\hline Diabetes mellitus & 7 (23.3\%) & $3(10.0 \%)$ & $5(16.7 \%)$ & 0.383 \\
\hline History of abdominal surgery & $7(23.3 \%)$ & $4(13.3 \%)$ & $6(20.0 \%)$ & 0.602 \\
\hline \multicolumn{5}{|l|}{ Laboratory variables } \\
\hline White blood cell count $\left(\times 10^{9} / \mathrm{L}\right)$ & $5.4(4.6-7.4)$ & $7.1(5.9-7.6)$ & $6.2(5.2-7.5)$ & 0.133 \\
\hline Neutrophil (\%) & $54.3(47.9-57.2)$ & $55.1(51.6-59.6)$ & $52.9(51.1-54.5)$ & 0.118 \\
\hline Lymphocyte (\%) & $33.8(31.3-38.3)$ & $32.6(31.1-40.4)$ & $36.5(34.6-39.0)$ & 0.089 \\
\hline Hemoglobin (g/dL) & $14.3(13.8-14.8)$ & $13.9(13.1-15.4)$ & $14.7(13.7-15.6)$ & 0.375 \\
\hline Platelet count $\left(\times 10^{9} / \mathrm{L}\right)$ & $187.0(160.5-221.0)$ & $193.0(169.8-242.0)$ & $207.0(172.3-232.3)$ & 0.52 \\
\hline International normalized ratio & $0.9(0.8-0.9)$ & $0.9(0.9-0.9)$ & $0.9(0.8-0.9)$ & 0.148 \\
\hline \multicolumn{5}{|l|}{ Intraoperative findings } \\
\hline Surgical duration (min) & $123(109-145)$ & $123(100-141)$ & $123(114-138)$ & 0.713 \\
\hline Hypotension event ${ }^{a}$ & $10(33.3 \%)$ & $15(50.0 \%)$ & $17(56.7 \%)$ & 0.175 \\
\hline Total rescue ephedrine infusion (mg) & $0(0-4)$ & $2(0-8)$ & $4(0-8)$ & 0.139 \\
\hline Total remifentanil infusion (mg) & $0.5(0.4-0.6)$ & $0.4(0.3-0.4)^{*}$ & $0.2(0.1-0.3)^{*,+}$ & $<0.001$ \\
\hline Crystalloid fluid infusion (mL) & $500(400-600)$ & $575(400-663)$ & $525(388-800)$ & 0.782 \\
\hline Urine output (mL) & $100(50-100)$ & $50(50-100)$ & $100(50-100)$ & 0.496 \\
\hline Hemorrhage $(\mathrm{mL})$ & $100(50-100)$ & $100(50-100)$ & $100(50-163)$ & 0.405 \\
\hline
\end{tabular}

Abbreviations: IV-PCA Intravenous patient-controlled analgesia, VAS Visual analog scale, PACU Post-anesthesia care unit ${ }^{*} p<0.017$ as statistical significance based on the level in the IV-PCA group ${ }^{+} p<0.017$ as statistical significance based on the level in the RSB group a Hypotension event defined as systolic blood pressure $<90 \mathrm{mmHg}$ over $10 \mathrm{~min}$ NOTE: Values are expressed as the median (interquartile) and number (proportion)

pruritus than RSB and IV-PCA alone, we noted no ITMB-related, postoperative adverse event, such as respiratory depression, lower leg numbness, or post-dural puncture headache.

Our ITMB data are similar to those of previous laparoscopic and open surgery reports [5, 22-24]; thus, ITMB is a feasible and practicable form of pain relief (yielding a lower pain score and lower opioid requirement). Moreover, it was not associated with serious complications (such as nerve injury) during or after surgery, and was better than RSB or IV-PCA alone. The differences between intrathecal and peripheral blocks include the sites affected by the analgesic drugs and subsequent drug actions. Intrathecally injected morphine and bupivacaine become widely dispersed in cerebrospinal fluid, thus more reliably (compared with RSB) preventing nociceptive inputs from multiple somatic dermatome levels in patients undergoing RALP [24, 25]. The principal skin wound created during laparoscopy- based surgery lies in the peri-umbilical area; the orifice is used for camera insertion and specimen (prostate mass) removal. In the past, RSB effectively countered pain caused by injury to the peri-umbilical dermatomes $[26,27]$. However, as surgery advanced from open surgery to human-executed laparoscopic surgery to RALP (reducing the operation time and numbers of painful stimuli delivered by surgical wounds in sites such as the umbilicus) [28], the analgesic effect of RSB seems to have gradually decreased along with improvements in surgical wound care techniques. Furthermore, ITMB may deliver visceral analgesia by interacting with spinal $\mu$ - and $\mathrm{k}$-opioid receptors and voltage-gated sodium channels that contain binding sites for local anesthetics. It is now possible to totally (and simultaneously) avoid the surgical stress and pain imparted by intra-abdominal wounds (created when prostate-adjacent tissues are dissected and retracted) and skin wounds (created when the skin is incised, punctured, and retracted) $[29,30]$. 


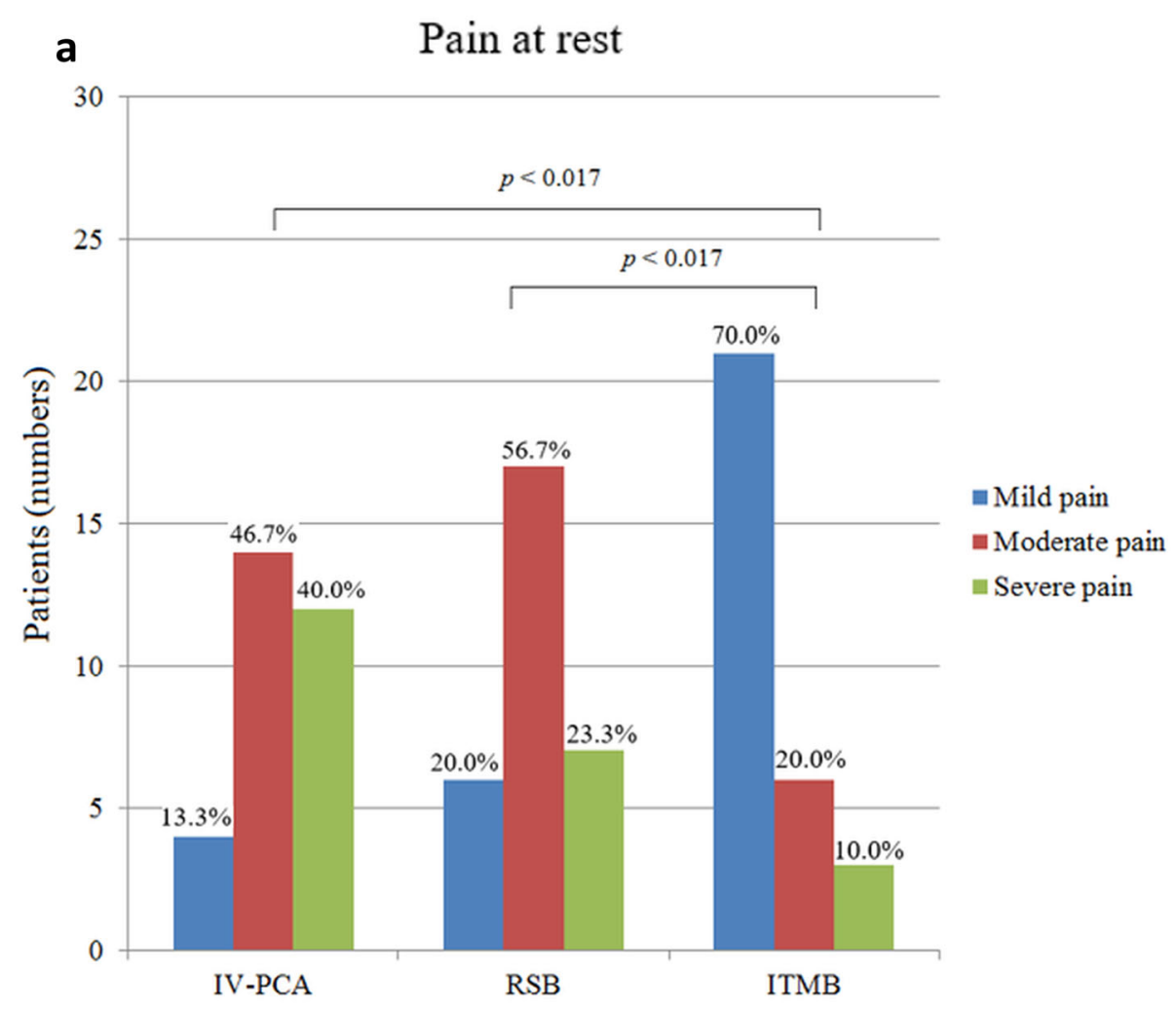

b

Pain at cough

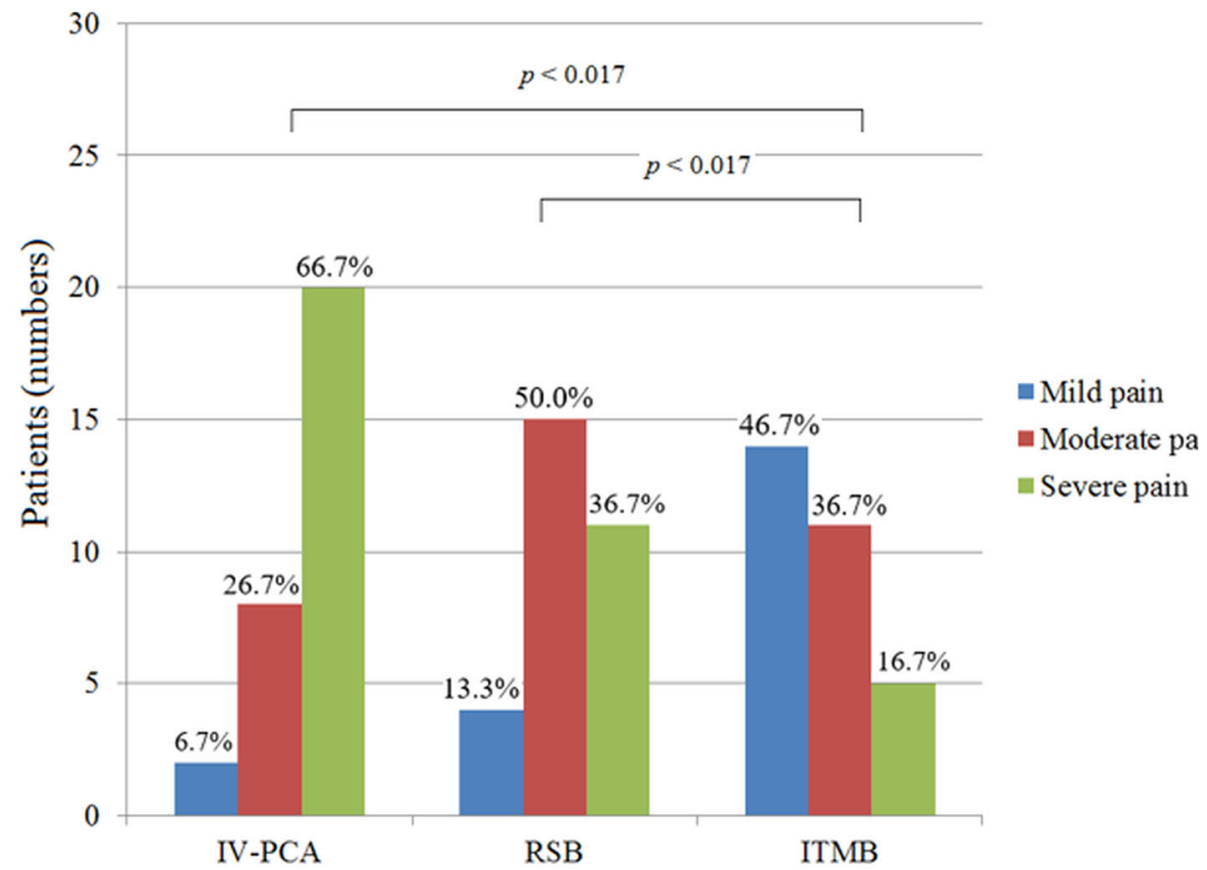

Fig. 3 Pain scores (a) at rest and (b) with coughing in the three groups ( $n=30$ per group) in the first $24 \mathrm{~h}$ postoperatively. Mild pain was defined as a peak VAS score of $0-3$, moderate pain as a peak score of 4-6, and severe pain as a peak score of 7-10. $p<0.017$ indicates statistical significance (adjusted for multiple comparisons). Values are expressed as numbers with proportions (\% values) 
Table 2 Analgesic efficacy of IV-PCA, the RSB, and ITMB block with severe pain (peak VAS $\geq 7$ ) at rest and cough during $24 \mathrm{~h}$ postoperatively

\begin{tabular}{|c|c|c|c|c|}
\hline & B & Odds ratio & 95\% Confidence interval & $p$ \\
\hline \multicolumn{5}{|c|}{ Severe pain at rest } \\
\hline \multicolumn{5}{|c|}{$\begin{array}{l}\text { Analgesia adjusted for age, BMI, DM, hypertension, and intraoperative } \\
\text { remifentanil consumption }\end{array}$} \\
\hline IV-PCA & \multicolumn{4}{|c|}{ Reference } \\
\hline RSB & -0.74 & 0.477 & $0.156-1.464$ & 0.196 \\
\hline ITMB & -1.792 & 0.167 & $0.041-0.675$ & 0.012 \\
\hline \multicolumn{5}{|c|}{ Severe pain at cough } \\
\hline \multicolumn{5}{|c|}{$\begin{array}{l}\text { Analgesia adjusted for age, BMI, DM, hypertension, and intraoperative } \\
\text { remifentanil consumption }\end{array}$} \\
\hline IV-PCA & \multicolumn{4}{|c|}{ Reference } \\
\hline RSB & -1.186 & 0.306 & $0.105-0.888$ & 0.029 \\
\hline ITMB & -2.303 & 0.1 & $0.029-0.34$ & $<0.001$ \\
\hline
\end{tabular}

Abbreviation: VAS Visual analog scale, IV-PCA Intravenous patient-controlled analgesia, $R S B$ Rectus sheath block, ITMB Intrathecal morphine with bupivacaine, $B M I$ Body mass index, DM Diabetes mellitus

However, RSB inhibits only somatic, afferent nerve pain. Thus, it cannot deliver comprehensive analgesia; pain from the visceral origins is not dulled [26].

In terms of complications, postoperative nausea/ vomiting and pruritus compromise the quality of patient recovery [31]. Previous studies suggested that the incidences of such complications were higher in patients who received intrathecal morphine than in those receiving local anesthetic-based analgesia [32-34]. Intrathecal block with low doses of combined morphine (75 or $100 \mu \mathrm{g})$ and bupivacaine $(5 \mathrm{mg}$ ) provided more effective postoperative analgesia than did sham subcutaneous block with normal saline. However, the side effects (e.g., nausea and vomiting) were of comparable incidence and severity between the two groups [35]. Our ITMB regimen included bupivacaine $(7.5 \mathrm{mg})$, which allowed us to reduce the morphine dose to $0.2 \mathrm{mg}$, thus reducing nausea/vomiting and pruritus while maintaining appropriate analgesia. However, we found higher incidences of nausea and pruritus in patients who received ITMB than in those who received RSB or IV-PCA alone. Notably, our incidences of nausea (30.0\%), vomiting (6.7\%), and pruritus $(16.7 \%)$ in the ITMB group may be lower than those of patients who receive intrathecal morphine (without additive bupivacaine). Specifically, the reported incidences of nausea/vomiting and pruritus after surgery were approximately $60-80 \%$ and $30-100 \%$, respectively [36-38]. Nguyen et al. [39] suggested that the addition of bupivacaine $(15 \mathrm{mg})$ to intrathecal morphine $(0.4 \mathrm{mg})$ improved pain relief and reduced the incidence of adverse events (such as hypotension) in patients undergoing laparoscopic liver resection. Girgin et al. [40] found that the incidence of pruritus increased as the dose of intrathecal morphine rose from 0.1 to $0.4 \mathrm{mg}$; however, when morphine was combined with low-dose bupivacaine $(7.5 \mathrm{mg})$, the complication rate was reduced while analgesia remained stable in women undergoing cesarean sections. The good analgesia (i.e., reduced requirement for IV opioid infusion) without adverse complications (Clavien-Dindo grade $\geq$ III), afforded by our low-dose ITMB regimen, may enhance early postoperative recovery compared with patients treated via RSB or IV-PCA alone.

In our study, there were comparable clinical characteristics, such as surgical duration, hypotension events, and total rescue ephedrine infusion, between the groups. Notably, analgesic treatments (RSB vs. ITMB) may not affect the prolongation of surgical time that possibly results from management by attending anesthesiologists who have excellent technique and sufficient experience with regional blocks, although there were differences in the RSB and ITMB regimens, such as target sites (rectus muscle vs. spine), needle manipulation (ultrasound-based vs. palpation-based), and patient position (supine vs. lateral) [41, 42]. Furthermore, intrathecal injection of bupivacaine may produce a high level of sensory and motor block, as well as arterial hypotension. However, these effects were dose-dependent, such that the lowest dose of bupivacaine (7 mg) provided equally rapid onset and effective anesthesia for cesarean surgery while reducing the incidence of hypotension, compared with bupivacaine doses of 8 and $9 \mathrm{mg}$, in patients who received combined administration of intrathecal morphine $(100 \mu \mathrm{g})$ [43]. These findings were consistent with ours in that a low dose of additive intrathecal bupivacaine $(7.5 \mathrm{mg})$ may have minimal impact on the occurrence of persistent hypotension and requirement for rescue inotrope.

Our work had certain limitations. First, we delivered single bupivacaine injections to the rectus sheath when

Table 3 Linear trend for cumulative IV-PCA drug consumption during POD 1 among the IV-PCA vs. RSB vs. ITMB groups

\begin{tabular}{ccc} 
Table 3 Linear trend for cumulative IV-PCA drug consumption during POD 1 among the N-PCA VS. RSB VS. ITMB groups \\
\hline $\mathbf{9 5}$ Confidence interval
\end{tabular}

Cumulative IV-PCA drug consumption during POD $1(\mathrm{~mL})$

Adjusted for age, BMI, DM, hypertension, and intraoperative remifentanil consumption 
Table 4 Comparisons of patient outcomes during the first $24 \mathrm{~h}$ postoperatively between the three groups

\begin{tabular}{|c|c|c|c|c|}
\hline Group & IV-PCA & RSB & ITMB & \\
\hline $\mathrm{n}$ & 30 & 30 & 30 & $p$ \\
\hline \multicolumn{5}{|l|}{ Requirement of opioid infusion } \\
\hline Cumulative IV-PCA infusion (mL) & $37.6(25.9-57.3)$ & $42.8(29.9-60.8)$ & $18.7(14.7-26.2)^{*},+$ & $<0.001$ \\
\hline IV rescue opioid infusion & $19(63.3 \%)$ & $21(70.0 \%)$ & $6(20.0 \%)^{*},+, \neq$ & $<0.001$ \\
\hline \multicolumn{5}{|l|}{ Peak visual analog scale } \\
\hline at rest & $6(4-7)$ & $5(4-6)$ & $3(2-4)^{*},+$ & $<0.001$ \\
\hline at cough & $8(6-9)$ & $6(5-7)^{*}$ & $4(3-5)^{*},+$ & $<0.001$ \\
\hline \multicolumn{5}{|l|}{ Quality of early recovery } \\
\hline Global score of QoR-15 questionnaire on POD 1 & $124(122-129)$ & $124(117-133)$ & $130(126-141)^{*},+$ & 0.002 \\
\hline \multicolumn{5}{|l|}{ Complications } \\
\hline Nausea & $2(6.7 \%)$ & $2(6.7 \%)$ & $9(30.0 \%)^{\ddagger}$ & 0.012 \\
\hline Vomiting & $0(0.0 \%)$ & $1(3.3 \%)$ & $2(6.7 \%)$ & 0.355 \\
\hline Pruritus & $0(0.0 \%)$ & $0(0.0 \%)$ & $5(16.7 \%)^{\ddagger}$ & 0.005 \\
\hline \multicolumn{5}{|l|}{ Laboratory variables on POD 1} \\
\hline White blood cell count $\left(\times 10^{9} / \mathrm{L}\right)$ & $13.5(8.7-16.5)$ & $15.4(8.2-20.7)$ & $14.3(11.2-19.3)$ & 0.478 \\
\hline Neutrophil (\%) & $172.0(141.0-203.3)$ & $175.5(154.0-211.8)$ & $168.0(151.3-201.0)$ & 0.873 \\
\hline Lymphocyte (\%) & $73.5(69.1-76.5)$ & $73.1(68.0-78.7)$ & $71.4(65.1-78.9)$ & 0.807 \\
\hline Hemoglobin (g/dL) & $8.8(7.4-11.0)$ & $7.8(7.1-9.1)$ & $8.7(6.6-10.3)$ & 0.476 \\
\hline Platelet count $\left(\times 10^{9} / \mathrm{L}\right)$ & $12.6(11.7-13.4)$ & $12.2(11.0-13.5)$ & $12.3(11.7-12.9)$ & 0.419 \\
\hline International normalized ratio & $1.0(0.8-1.0)$ & $0.9(0.9-1.0)$ & $0.9(0.8-1.0)$ & 0.072 \\
\hline
\end{tabular}

Abbreviations: QoR-15 Quality of Recovery-15 questionnaire, $P O D$ Postoperative day

${ }^{*} p<0.017$ as statistical significance based on the level in the IV-PCA group

${ }^{+} p<0.017$ as statistical significance based on the level in the RSB group

${ }^{\ddagger} p<0.05$ using the linear-by-linear method

NOTE: Values are expressed as the median (interquartile) and number (proportion)

comparing the outcomes of the three pain-relief regimens. However, no ideal regional analgesic technique for RALP has yet been established; other regional analgesic models, including catheter-delivered continuous blockade, may be superior to ITMB [44]. Second, because of the absence of robust evidence related to an acceptable range of INRs for single-injection ultrasound-guided subfascial block (i.e., RSB), we presumed that an INR $>2.0$ was the highest acceptable level for inclusion in our study. However, because of the risk of hematoma, skillful and meticulous ultrasound-based RSB is required for patient safety and analgesic results. Further RSB analyses are needed to determine the acceptable ranges of coagulopathic parameters, such as the INR. Third, our study was limited in that patients were not randomly allocated, despite the presence of comparable groups. A randomized setting was considered but rejected due to ethical concerns that IV-PCA alone may provide insufficient pain relief, compared with the other two analgesic regimens. Therefore, it was not possible to determine whether the analgesic results were solely related to pain-relief regimens.

\section{Conclusions}

ITMB may usefully reduce postoperative pain and aid recovery in patients undergoing RALP. Although robotassisted surgery is more advanced and less invasive than open or laparoscopic surgery, analgesic care must counter both parietal and visceral pain associated with multilevel skin wounds and intra-abdominal tissue injuries. Our ITMB regimen (a low dose of morphine [0.2 mg] combined with bupivacaine $[7.5 \mathrm{mg}]$ ) may contribute superior analgesia and better patient perception in terms of early postoperative recovery.

\section{Supplementary Information}

The online version contains supplementary material available at https:/doi. org/10.1186/s12871-020-01208-2.

Additional file 1. "Strengthening the Reporting of Observational Studies in Epidemiology" (STROBE) guidelines.

\section{Abbreviations}

RALP: Robot-assisted laparoscopic prostatectomy; RSB: Rectus sheath block; TAP: Trasnversus abdominis plane block; ITMB: Intrathecal morphine and bupivacaine block; POD: Postoperative day; IV-PCA: Intravenous patientcontrolled analgesia; ASA: American Society of Anesthesiologists; 
INR: Interntaional normalized ratio; VAS: Visual analogue scale; QoR15: Quality-of-recovery score on a 15-item questionnaire

\section{Acknowledgements}

The authors would like to thank our anesthetic nursing team (Eunju Choi and Hyeji An; Seoul St. Mary's Hospital, The Catholic University of Korea, Seoul, Republic of Korea) for their dedication.

\section{Authors' contributions}

J.W.S. and M.S.C. designed the study, wrote the manuscript, and analyzed and interpreted the data. J.W.S., Y.J.C., M.K., S.H.H., H.W.M., S.H.H. and M.S.C. collected the data and provided critical comments. All authors revised the manuscript critically for important intellectual content. All authors read and approved the final manuscript.

\section{Funding}

No funding was used for this work.

\section{Availability of data and materials}

The datasets used and/or analyzed during this study are available from the corresponding author on reasonable request.

\section{Ethics approval and consent to participate}

This was a prospective, observational parallel-cohort trial. The protocol was approved by the Institutional Review Board of Seoul St. Mary's Hospital Ethics Committee (approval no. KC20OISI0124) on April 29, 2020. The study was performed in accordance with all relevant principles of the Declaration of Helsinki. The study protocol was prospectively registered on a publicly accessible clinical registration site recognized by the International Committee of Medical Journal Editors (Clinical Research Information Service, Republic of Korea; approval no. KCT0005040) on May 20, 2020. Written informed consent was obtained from all patients enrolled between May and July, 2020. The study adhered to Strengthening the Reporting of Observational Studies in Epidemiology guidelines.

\section{Consent for publication}

Not applicable.

\section{Competing interests}

The authors have no conflicts of interest to declare.

\section{Author details}

${ }^{1}$ Department of Anesthesiology and Pain medicine, Seoul St. Mary's Hospital, College of Medicine, The Catholic University of Korea, 222, Banpo-daero, Seocho-gu, Seoul 06591, Republic of Korea. '2Department of Anesthesiology and Pain medicine, Bucheon St. Mary's Hospital, College of Medicine, The Catholic University of Korea, Seoul, Republic of Korea. ${ }^{3}$ Department of Urology, Seoul St. Mary's Hospital, College of Medicine, The Catholic University of Korea, Seoul, Republic of Korea.

\section{Received: 25 August 2020 Accepted: 17 November 2020}

\section{Published online: 23 November 2020}

\section{References}

1. Nossiter J, Sujenthiran A, Charman SC, Cathcart PJ, Aggarwal A, Payne H, et al. Robot-assisted radical prostatectomy vs laparoscopic and open retropubic radical prostatectomy: functional outcomes 18 months after diagnosis from a national cohort study in England. Br J Cancer. 2018;118: 489-94.

2. Pearce SM, Pariser JJ, Karrison T, Patel SG, Eggener SE. Comparison of perioperative and early oncologic outcomes between open and robotic assisted laparoscopic prostatectomy in a contemporary population based cohort. J Urol. 2016:196:76-81.

3. Porpiglia F, Fiori C, Bertolo R, Manfredi M, Mele F, Checcucci E, et al. Fiveyear outcomes for a prospective randomised controlled trial comparing laparoscopic and robot-assisted radical prostatectomy. Eur Urol Focus. 2018; 4:80-6.

4. Woldu SL, Weinberg AC, Bergman A, Shapiro EY, Korets R, Motamedinia P, et al. Pain and analgesic use after robot-assisted radical prostatectomy. J Endourol. 2014;28:544-8.
5. Koning MV, de Vlieger R, Teunissen AJW, Gan M, Ruijgrok EJ, de Graaff JC, et al. The effect of intrathecal bupivacaine/morphine on quality of recovery in robot-assisted radical prostatectomy: a randomised controlled trial. Anaesthesia. 2019:75:599-608.

6. Dal Moro F, Aiello L, Pavarin P, Zattoni F. Ultrasound-guided transversus abdominis plane block (US-TAPb) for robot-assisted radical prostatectomy: a novel '4-point' technique-results of a prospective, randomized study. J Robot Surg. 2019;13:147-51.

7. Gupta M, Naithani U, Singariya G, Gupta S. Comparison of $0.25 \%$ Ropivacaine for Intraperitoneal instillation $\mathrm{v} / \mathrm{s}$ rectus sheath block for postoperative pain relief following laparoscopic cholecystectomy: a prospective study. J Clin Diagn Res. 2016;10:Uc10.

8. Yasumura R, Kobayashi $Y$, Ochiai R. A comparison of plasma levobupivacaine concentrations following transversus abdominis plane block and rectus sheath block. Anaesthesia. 2016:71:544-9.

9. Bae J, Kim HC, Hong DM. Intrathecal morphine for postoperative pain control following robot-assisted prostatectomy: a prospective randomized trial. J Anesth. 2017;31:565-71.

10. Buyyounouski MK, Choyke PL, McKenney JK, Sartor O, Sandler HM, Amin MB, et al. Prostate cancer - major changes in the American joint committee on cancer eighth edition cancer staging manual. CA Cancer J Clin. 2017:67: 245-53.

11. Horlocker TT, Vandermeuelen E, Kopp SL, Gogarten W, Leffert LR, Benzon HT. Regional anesthesia in the patient receiving antithrombotic or thrombolytic therapy: American Society of Regional Anesthesia and Pain Medicine evidence-based guidelines (fourth edition). Reg Anesth Pain Med. 2018;43:263-309.

12. Sites BD, Chan WW, Neal JM, Weller R, Grau T, Koscielniak-Nielsen ZJ, et al The American Society of Regional Anesthesia and Pain Medicine and the European Society of Regional Anaesthesia and Pain Therapy joint committee recommendations for education and training in ultrasoundguided regional anesthesia. Reg Anesth Pain Med. 2010;35:S74-80.

13. American Society of Anesthesiologists Task Force on Perioperative Blood Management. Practice guidelines for perioperative blood management: an updated report by the American Society of Anesthesiologists Task Force on perioperative blood management*. Anesthesiology. 2015:122:241-75.

14. Shim JW, Moon HK, Park YH, Park M, Park J, Lee HM, et al. Intraoperative changes in whole-blood viscosity in patients undergoing robot-assisted laparoscopic prostatectomy in the steep Trendelenburg position with pneumoperitoneum: a prospective nonrandomized observational cohort study. BMC Anesthesiol. 2020;20:7.

15. Hawker GA, Mian S, Kendzerska T, French M. Measures of adult pain: visual analog scale for pain (VAS pain), numeric rating scale for pain (NRS pain), McGill pain questionnaire (MPQ), short-form McGill pain questionnaire (SFMPQ), chronic pain grade scale (CPGS), short Form-36 bodily pain scale (SF$36 \mathrm{BPS})$, and measure of intermittent and constant osteoarthritis pain (ICOAP). Arthritis Care Res. 2011:63(Suppl 11):S240-52.

16. Stark PA, Myles PS, Burke JA. Development and psychometric evaluation of a postoperative quality of recovery score: the QoR-15. Anesthesiology. 2013; 118:1332-40.

17. Clavien PA, Barkun J, de Oliveira ML, Vauthey JN, Dindo D, Schulick RD, et al. The Clavien-Dindo classification of surgical complications: five-year experience. Ann Surg. 2009;250:187-96.

18. de Souza DS, Costa AF, Chaves GV. Predisposing factors for postoperative nausea and vomiting in gynecologic tumor patients. Support Care Cancer. 2016;24:4661-7.

19. Lockington PF, Fa'aea P. Subcutaneous naloxone for the prevention of intrathecal morphine induced pruritus in elective caesarean delivery. Anaesthesia. 2007:62:672-6.

20. Feldman EL, Nave KA, Jensen TS, Bennett DLH. New horizons in diabetic neuropathy: mechanisms, bioenergetics, and pain. Neuron. 2017:93:1296-313.

21. Bruehl S, Chung OY, Diedrich L, Diedrich A, Robertson D. The relationship between resting blood pressure and acute pain sensitivity: effects of chronic pain and alpha-2 adrenergic blockade. J Behav Med. 2008;31:71-80.

22. Boisen ML, McQuaid AJ, Esper SA, Holder-Murray J, Zureikat AH, Hogg ME, et al. Intrathecal morphine versus nerve blocks in an enhanced recovery pathway for pancreatic surgery. J Surg Res. 2019:244:15-22.

23. Tang $Y$, Tang $X$, Wei $Q$, Zhang $H$. Intrathecal morphine versus femoral nerve block for pain control after total knee arthroplasty: a meta-analysis. J Orthop Surg Res. 2017;12:125.

24. Lui MW, Li TKT, Lui F, Ong CYT. A randomised, controlled trial of rectus sheath bupivacaine and intrathecal bupivacaine, without or with 
intrathecal morphine, vs. intrathecal bupivacaine and morphine after caesarean section. Anaesthesia. 2017;72:1225-9.

25. Jeong HW, Kim CS, Choi KT, Jeong SM, Kim DH, Lee JH. Preoperative versus postoperative rectus sheath block for acute postoperative pain relief after laparoscopic cholecystectomy. a randomized controlled study. J Clin Med. 2019;8:1018.

26. Karaarslan E, Topal A, AvcI O, Tuncer US. Research on the efficacy of the rectus sheath block method. Agri. 2018;30:183-8.

27. Cockrell R, Bonzo J, Lee D. Robot-assisted simple prostatectomy. J Endourol. 2018;32:S33-s8.

28. Du Y, Long Q, Guan B, Mu L, Tian J, Jiang Y, et al. Robot-assisted radical prostatectomy is more beneficial for prostate cancer patients: a system review and meta-analysis. Med Sci Monit. 2018;24:272-87.

29. Stein C. New concepts in opioid analgesia. Expert Opin Investig Drugs. 2018;27:765-75.

30. Yanagidate F, Strichartz GR. Local anesthetics. Handb Exp Pharmacol. 2007: 95-127.

31. Ljungqvist O. ERAS--enhanced recovery after surgery: moving evidencebased perioperative care to practice. JPEN J Parenter Enteral Nutr. 2014;38: 559-66.

32. Kang R, Chin KJ. Bilateral single-injection erector spinae plane block versus intrathecal morphine for postoperative analgesia in living donor laparoscopic hepatectomy: a randomized non-inferiority trial; 2019.

33. Jun JH, Kim GS, Lee JJ, Ko JS, Kim SJ, Jeon PH. Comparison of intrathecal morphine and surgical-site infusion of ropivacaine as adjuncts to intravenous patient-controlled analgesia in living-donor kidney transplant recipients. Singap Med J. 2017:58:666-73.

34. De Pietri L, Siniscalchi A, Reggiani A, Masetti M, Begliomini B, Gazzi M, et al. The use of intrathecal morphine for postoperative pain relief after liver resection: a comparison with epidural analgesia. Anesth Analg. 2006;102 1157-63.

35. Motamed C, Bouaziz H, Franco D, Benhamou D. Analgesic effect of lowdose intrathecal morphine and bupivacaine in laparoscopic cholecystectomy. Anaesthesia. 2000;55:118-24

36. Koju RB, Gurung BS, Dongol Y. Prophylactic administration of ondansetron in prevention of intrathecal morphine-induced pruritus and post-operative nausea and vomiting in patients undergoing caesarean section. BMC Anesthesiol. 2015:15:18.

37. Kumar K, Singh SI. Neuraxial opioid-induced pruritus: an update. J Anaesthesiol Clin Pharmacol. 2013;29:303-7.

38. Szarvas S, Chellapuri RS, Harmon DC, Owens J, Murphy D, Shorten GD. A comparison of dexamethasone, ondansetron, and dexamethasone plus ondansetron as prophylactic antiemetic and antipruritic therapy in patients receiving intrathecal morphine for major orthopedic surgery. Anesth Analg. 2003;97:259-63 table of contents.

39. Nguyen M, Vandenbroucke F, Roy JD, Beaulieu D, Seal RF, Lapointe R, et al. Evaluation of the addition of bupivacaine to intrathecal morphine and fentanyl for postoperative pain management in laparascopic liver resection. Reg Anesth Pain Med. 2010;35:261-6.

40. Girgin NK, Gurbet A, Turker G, Aksu H, Gulhan N. Intrathecal morphine in anesthesia for cesarean delivery: dose-response relationship for combinations of low-dose intrathecal morphine and spinal bupivacaine. J Clin Anesth. 2008;20:180-5.

41. Kim SC, Hauser S, Staniek A, Weber S. Learning curve of medical students in ultrasound-guided simulated nerve block. J Anesth. 2014;28:76-80.

42. Baranauskas MB, Margarido CB, Panossian C, Silva ED, Campanella MA, Kimachi PP. Simulation of ultrasound-guided peripheral nerve block: learning curve of CET-SMA/HSL anesthesiology residents. Rev Bras Anestesiol. 2008:58:106-11.

43. Leo S, Sng BL, Lim Y, Sia AT. A randomized comparison of low doses of hyperbaric bupivacaine in combined spinal-epidural anesthesia for cesarean delivery. Anesth Analg. 2009;109:1600-5.

44. Álvarez NER, Ledesma RJG, Hamaji A, Hamaji MWM, Vieira JE. Continuous femoral nerve blockade and single-shot sciatic nerve block promotes better analgesia and lower bleeding for total knee arthroplasty compared to intrathecal morphine: a randomized trial. BMC Anesthesiol. 2017:17:64

\section{Publisher's Note}

Springer Nature remains neutral with regard to jurisdictional claims in published maps and institutional affiliations.

\section{Ready to submit your research? Choose BMC and benefit from}

- fast, convenient online submission

- thorough peer review by experienced researchers in your field

- rapid publication on acceptance

- support for research data, including large and complex data types

- gold Open Access which fosters wider collaboration and increased citations

- maximum visibility for your research: over $100 \mathrm{M}$ website views per year

At $\mathrm{BMC}$, research is always in progress.

Learn more biomedcentral.com/submissions 